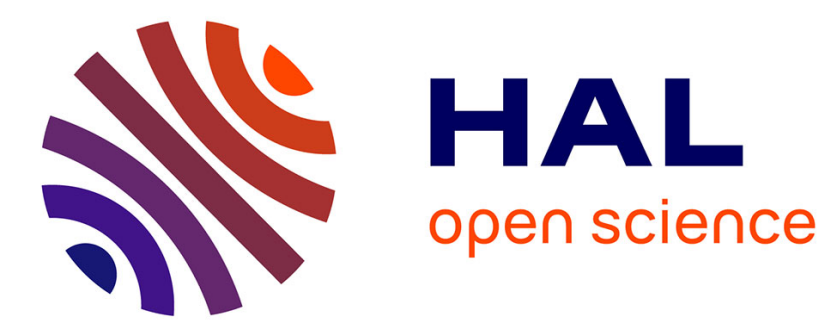

\title{
Recent breakthroughs in nickel base superalloys Y. Honnorat
}

\section{To cite this version:}

Y. Honnorat. Recent breakthroughs in nickel base superalloys. Journal de Physique IV Proceedings, 1993, 03 (C7), pp.C7-297-C7-323. 10.1051/jp4:1993747 . jpa-00252165

\section{HAL Id: jpa-00252165 https://hal.science/jpa-00252165}

Submitted on 1 Jan 1993

HAL is a multi-disciplinary open access archive for the deposit and dissemination of scientific research documents, whether they are published or not. The documents may come from teaching and research institutions in France or abroad, or from public or private research centers.
L'archive ouverte pluridisciplinaire HAL, est destinée au dépôt et à la diffusion de documents scientifiques de niveau recherche, publiés ou non, émanant des établissements d'enseignement et de recherche français ou étrangers, des laboratoires publics ou privés. 


\title{
Recent breakthroughs in nickel base superalloys
}

\author{
Y. HONNORAT
}

SNECMA, General Manager Materials and Processes

\begin{abstract}
Meanwhile the considerable amount of results acquired since more than sixty years in the study of this class of materials, the pre-eminence of nickel base superalloys in the gas turbine engineering, which is a domain in constant evolution, drives the significant progresses accomplished along the five last years. The knowledge, each day more precisely known, of the working conditions of the parts, the continuous increase of the computer capacity and the progressive sophistication of the designing methods end up in very high levels of requirements, progressively more complex, which induce the major routes of development. The particular accomplishments in three different domains of application will be described in this paper.

Significant increases of performance have been acquired on directionally solidified turbine blades by an in-depth investigation of strain and damage mechanisms. Behaviour and ruin models take more and more into account the physical reality at each scale of observation of the crystalline structure response to coupled prompting of creep, fatigue and oxidation. On such a basis, a judicious choice of analytical compositions and overlays, with adapted conditions of manufacture enhance the use capability of the parts.

In an other domain, decisive advantage has been taken of the variability of the forged materials devoted to disc applications. From the same chemistry of alloy, it is possible to optimize the structures according to various combinations of local stressing. This approach is funded on the disposal of efficient methods of microstructure prediction in relationship with the local rheological history. Precise control methods taking into account the complexity of the resulting microstructure allow the production of parts associated with narrow limits of life duration. At last, shall be discussed the significance of an adequate control of inclusion distribution and cleanliness in highly stressed superalloys in relationship with fatigue life duration. The problem more specific to superalloys produced by prealloyed powder metallurgy routes shall be analyzed and the solutions adapted to civil engine applications exposed.
\end{abstract}




\section{PROGRES RECENTS DANS LES SUPERALLIAGES DE NICKEL}

\section{INTRODUCTION}

Il est reconnu depuis soixante ans que les superalliages de nickel, représentant la famille des alliages métalliques fondés sur le système ternaire nickel-chrome-aluminium combinent des propriétés exceptionnelles de résistance mécanique, de tenacité et de tenue à l'oxydation depuis les plus basses températures jusqu'au delà de $1100^{\circ} \mathrm{C}$, plus près de leur point de fusion qu'aucune autre classe de matériaux métalliques [1]. Cette combinaison exceptionnelle de caractéristiques est due à trois facteurs essentiels :

- l'absence de transformation allotropique de leur matrice cubique à face centrée, qui offre de multiples possibilités de glissements,

- la stabilité jusqu'à la fusion de l'ordre à longue distance de la phase durcissante $\gamma^{\text {' de }}$ structure cubique $\mathrm{Ll}_{2}$,

- la faible cinétique d'oxydation associée à une couche d'oxyde solide adhérente au substrat et relativement imperméable (oxyde de chrome à basse température, alumine à haute température).

Malgré une densité double de celle des alliages d'aluminium et une résistance moitié de celle des aciers, les particularités des superalliages de nickel en font des matériaux structuraux irremplaçables dans beaucoup de domaines de la construction mécanique, en particulier celle des moteurs aéronautiques, où les familles d'alliages et les techniques de mise en oeuvre se sont progressivement différenciées en vue d'une meilleure adaptation aux exigences fonctionnelles des concepteurs. Les progrès continus en consommation de kérosène, en compacité, en légèreté des moteurs aéronautiques sont aujourd'hui fondés sur ceux des matériaux et des technologies de fabrication, et de ce fait les travaux de recherche et de développement dans le domaine des superalliages sont et restent considérables, comme l'atteste par exemple tous les quatre ans la vitalité du congrès de Seven-Spring [2] et l'intérêt qu'il suscite.

Pour illustrer les récents progrès de la recherche dans le domaine des superalliages et leur lien étroit avec des thèmes de développement des moteurs aéronautiques, nous décrivons ci-après les résultats acquis dans plusieurs cercles de coopération que SNECMA a suscités en France pour répondre à ses besoins. C'est ainsi que nous évoquerons successivement les acquis les plus récents concernant les superalliages monocristallins utilisés pour les aubes de turbine, la mise en oeuvre des superalliages coulés puis forgés pour disques annulaires de turbine à basse pression, et enfin l'endommagement et la ruine des superalliages de la métallurgie des poudres préalliées utilisés pour les disques de corps à haute pression.

\section{SUPERALLIAGES MONOCRISTALLINS}

Les premiers superalliages monocristallins dérivaient directement des compositions mises au point pour des pièces coulées à structure équiaxe, par suppression des éléments renforçateurs des joints de grains, en l'occurrence le bore, le carbone, le zirconium et l'hafnium. Ces alliages se caractérisaient généralement après homogénéisation par la présence de zones résiduelles d'hétérogénéités à structure lamellaire $\gamma / \gamma^{\prime}$ limitant les températures maximales d'emploi et la 
proportion acceptable de phase $\gamma^{\prime}$ durcissante. Outre que ces hétérogénéités structurales favorisent la propension à la recristallisation locale lors des traitements thermiques, elles entraînent aussi des limitations sur les propriétés de fluage et de fatigue. En même temps qu'elle développait un alliage AM 1 répondant à ses besoins, SNECMA a fédéré avec ses partenaires industriels et les Agences Gouvernementales concernées un nombre important de laboratoire de recherche dans trois Groupements Scientifiques successifs pour approfondir les connaissances dans tous les domaines reliant la composition à la microstructure et au comportement en service des aubes monocristallines.

\section{Composition et microstructure}

Pour ce qui concerne les relations entre la composition et la microstructure, il n'est pas inutile de rappeler que $M$. Durand-Charre et son équipe avaient établi en 1988 [3] que la nature de l'équilibre entre $\gamma, \gamma^{\prime}$ et le liquide, qui est de type eutectique dans le binaire $\mathrm{Ni} / \mathrm{Al}$, change de nature le long de la ligne monovariante des diagrammes multi-éléments, en particulier avec les additions de titane et de tantale pour devenir péritectique (cf. l'exemple de la figure 1). Cette analyse dont il serait trop long de rappeler ici le détail permet d'expliquer le désavantage de certains champs de composition aboutissant par refroidissement lent à la précipitation en fin de solidification de phases riches en chrome, tungstène, titane ou tantale formant des barrières de diffusion handicapantes lors des cycles d'homogénéisation. L'alliage AM 1 qui commence sa solidification dans le domaine $\gamma$ ne souffre pas de cette particularité.

Dans les mêmes laboratoires de l'Institut National Polytechnique de Grenoble, R. Bonnet et son équipe ont, depuis [4], identifié les différentes configurations de fautes de surstructure et mesuré les énergies de dissociation correspondantes en fonction de la composition dans le système $\mathrm{Ni}$ - $\mathrm{Al}$ - $\mathrm{Ti}$ - $\mathrm{Cr}$, en vue d'expliquer l'influence de la composition sur l'anomalie de limite élastique des superalliages.

Nos connaissances sur la partition des éléments d'alliage entre la matrice et les précipités sont devenues très précises grâce aux travaux de $\mathrm{D}$. Blavette [5] à la sonde atomique de l'Université de Rouen. Il est maintenant acquis que les transitions entre $\gamma$ et $\gamma^{\prime}$ s'effectuent sur un plan atomique et que la distribution du chrome et du molybdène s'ordonne à courte distance dans $\gamma$ selon une structure cristallographique $\mathrm{DO}_{22}$ de type $\mathrm{Ni}_{3} \mathrm{Cr}$. Cet ordre à courte distance, qui persiste au moins jusqu'à $750^{\circ} \mathrm{C}$, affecte directement la structure et le comportement des dislocations. Par ailleurs des bandes de matrice enrichies en chrome et appauvries en nickel, de l'ordre de $100 \AA$ d'épaisseur sont mises en évidence autour des précipités $\gamma$ '.

A une échelle plus grande, l'étude de topographie par diffraction de rayonnement $\mathrm{X}$ synchroton d'éprouvettes multibranches de type représenté à la figure 2 a permis à N.Siredey et J.Lacaze[6] la description de la structure en sous -grains du squelette dendritique. La précipitation de $\gamma^{\prime}$ en arrière du front de solidification développe de fortes contraintes permettant d'expliquer la courbure des réseaux dans les sous-grains et leur progressive désorientation mutuelle. Parallèlement à ces travaux, la diffraction des neutrons a permis à A.Royer et P.Bastie [7] du Laboratoire de Spectrométrie Physique de Grenoble d'accéder à une mesure précise de la fraction volumique de $\gamma^{\prime}$ (figure 3), et, par diffraction des rayons $\gamma$, de préciser, après les travaux de A. Ati [8] et de D. Bellet [9] la tétragonalité de la phase $\gamma^{\prime}$ et la distorsion tétragonale de la phase $\gamma$. En outre, la diffusion des neutrons aux petits angles leur a permis de relier ces paramètres à l'évolution morphologique des précipités. Le moteur principal 
de la coalescence parait lié à l'existence de faibles gradients chimiques à l'intérieur des dendrites de solidification, gradients qui subsistent malgré les traitements thermiques de stabilisation des phases. Elle est accompagnée de flux anisotropes d'espèces chimiques, qui transforment les symétries cubiques initiales des deux phases $\gamma$ et $\gamma^{\prime}$ en symétries quadratiques. Cette diffusion produit, de façon concomitante, une micro-morphologie dite "en radeau" des deux phases (figure 4). Les écarts paramétriques des phases (mismatch) ont des valeurs différentes suivant une direction parallèle ou perpendiculaire aux radeaux en question. Ces écarts dépendent beaucoup de la forme générale des précipités. Les dislocations à la frontière des deux phases ont un rôle prédominant dans ces mécanismes de diffusion assistés par la température. Cette corrélation de la morphologie et de la cristallographie des phases est essentielle pour optimiser la tenue en fluage à haute température par un choix judicieux de composition chimique et de traitement thermique. L'analyse des spectres de Fourier issus du traitement d'images de la distribution morphologique et spatiale de la phase $\gamma^{\prime}$ permet de multiplier à moindre frais les observations fondamentales rapportées ci-dessus. C'est ainsi que $\mathbf{R}$. Tintillier [10] a montré récemment que la coalescence de la phase $\gamma^{\prime}$, sous contrainte ou non, obéit à une loi unique et caractéristique de la composition du matériau dérivée d'un modèle classique de type LifshitzSlyozov-Wagner (Figure 5) dont l'application permet de régler les séquences de traitement thermique et facilite l'expertise de pièces usagées.

\section{Mécanismes de déformation}

En parallèle à ces descriptions quantifiées de la microstructures des superalliages monocristallins et de leur évolution, s'est édifiée une interprétation très affinée des mécanismes de déformation sur la base d'observations microscopiques menées dans de nombreux laboratoires. La microscopie électronique en champ faible associée à la simulation de contraste des structures de dislocation s'est avérée sur la proposition de R. Bonnet [11] un outil puissant pour analyser les modes extrêmement complexes de mouvement des dislocations en fonction de la température, et de l'énergie en jeu dans la déformation. Ces travaux ont amené à remettre partiellement en cause et à enrichir les modèles antérieurs proposés par B. Kear et son équipe dans les années soixante pour arriver à comprendre, en fonction des conditions expérimentales, les transitions entre les modes de déplacement des dislocations de matrice et les énergies d'activation associées aux déformations mesurées à l'échelle macroscopique. C'est ainsi que B. Descamps et $\mathrm{M}$. Condat ont montré [12] que le cisaillement des précipités s'effectue en associant les trois types de défauts plans extrinsèques, intrinsèques et de paroi d'antiphase selon des séquences dépendant du sens de la déformation (traction ou compression), ce qui rend compte des consolidations en fatigue, et que ce mode complexe de déformation s'étend jusqu'à $950^{\circ} \mathrm{C}$. Ce schéma est confirmé à quelques variantes près par $\mathrm{M}$. Ignat [13]. Par ailleurs, $\mathrm{N}$. Clément [14] a montré, par des essais de déformation in-situ de la phase $\gamma^{\prime}$, que les mécanismes qui gouvernent cette déformation sont liés à des variations de la structure de coeur des dislocations. En dessous de $760^{\circ} \mathrm{C}$, la déformation est régulée par des changements locaux de configuration de coeur sessile/glissile, et, au dessus de cette température, le glissement visqueux de superdislocations se généralise. Ces glissements peuvent être soit octaédriques (111) soit cubiques (OO1). Selon les alliages, le glissement cubique commence à plus ou moins haute température et ceci peut justifier des règles de choix de composition.

Cependant ce type de relation composition/résistance au glissement n'est pas facile à gérer dans la perspective du choix d'une composition plus performante, compte-tenu des autres conséquences des modifications de composition. C'est ainsi que la figure 6 montre que l'intérêt d'une addition de rhénium n'est, au seul plan technique, pas évident, bien qu'il augmente la 
résistance au fluage. En effet, les meilleurs alliages sans rhénium offrent un gain de densité qui compense en partie une capacité en température un peu plus limitée, et seule l'appréciation des performances de l'ensemble de l'étage (disque + aubes) permet d'effectuer un choix judicieux..

\section{Résistance aux agressions chimiques et endommagement}

La tenue des aubes à la corrosion et à l'oxydation cyclique constitue aussi un facteur de préoccupation influant sur le choix du matériau. Compte tenu des températures de travail et des atmosphères au contact des pièces, les superalliages monocristallins n'offrent pas une résistance suffisante aux agressions chimiques rencontrées dans les moteurs. Une telle résistance passe par une capacité à développer en surface une couche protectrice adhérente à base d'alumine, et c'est en fait un couple alliage/revêtement qu'il faut sélectionner en veillant à la compatibilité mécanique et chimique des constituants.

La famille des revêtements fondés sur NiAl se révèle particulièrement efficace et les solutions développées par SNECMA et l'ONERA ont permis des adaptations très satisfaisantes. En fait, une bonne adaptation du revêtement NiAl à l'alliage passe comme l'ont montré $\mathrm{S}$. Alpérine et R. Morbioli [15] par des additions d'éléments faiblement oxydables comme le platine, le chrome ou le palladium qui permettent de gagner un facteur 2 à 4 sur la tenue à l'oxydationcorrosion. Du point de vue du comportement mécanique il est tout d'abord nécessaire de s'assurer d'une ductilité suffisante du revêtement dans le domaine de température de plastification en service du superalliage. La courbe de la figure 7 montre que le revêtement C1A mis au point par SNECMA, obtenu par chrome-aluminisation, est, de ce point de vue, satisfaisant.

L'essai de fatigue anisotherme développé à l'Ecole des Mines de Paris par R. Rémy et son équipe permet d'analyser plus finement les phénomènes [16]. C'est ainsi, en particulier que E.Chataigner [17] a mis en évidence l'intérêt, illustré à la figure 8 des revêtements multicouches tels que le $\mathrm{ClA}$, où la bifurcation à l'interface entre la couche externe de NiAl et la couche intermédiaire d'eutectoïde $\mathrm{NiAl} / \alpha \mathrm{Cr}$ des fissures amorcées en surface constitue un mécanisme très efficace de dissipation d'énergie, retardateur d'endommagement. II a montré aussi que les meilleurs revêtements se caractérisent par une plastification de celui-ci selon un mode proche de celui du substrat, pour éviter des boursouflements générateurs d'endommagements locaux précoces. La figure 9 montre, sur une éprouvette tubulaire monocristalline d'AM1 soumise à un chargement cyclique couplé de température et de contrainte, l'efficacité d'un tel revêtement $\mathrm{C} 1 \mathrm{~A}$, dans le domaine des déformations plastiques rencontrées en service, où le bénéfice est un facteur xlO sur la durée de vie.

On obtient par ailleurs une meilleure adhésion de la couche d'oxyde sur l'alliage par des additions mesurées $\left(10^{-4}\right.$ à $10^{-3}$ en poids) d'éléments avides d'oxygène des colonnes III et IV de la classification de Mendeleïev. Dans tous les cas, c'est l'addition d'yttrium qui a permis d'améliorer de façon la plus significative la tenue à l'oxydation des superalliages monocristallins. Cependant la réactivité de cet élément vis à vis des creusets d'élaboration, sa faible solubilité dans le nickel et sa forte ségrégation à la solidification sont la cause de rendements aléatoires et d'une réduction significative de la température de brûlure $[18,19]$. Pour contourner ces problèmes P.Caron a étudié [2O] à l'ONERA l'effet conjugué de l'addition simultanée de traces de plusieurs éléments. La meilleure combinaison s'avère être l'addition conjointe de silicium et de hafnium qui, à la concentration de $1000 \mathrm{ppm}$ en poids de chacun de ces deux éléments permet de gagner, à $1100^{\circ} \mathrm{C}$, un facteur d'au moins 10 sur la perte de masse de l'AM1 en oxydation cyclée, sans que des inconvénients liés à l'yttrium n'aient été rencontrés. 


\section{Modélisation du comportement}

Le dimensionnement des aubes impose l'écriture de lois de comportement monotone et cyclique du matériau anisotrope sous les trois aspects élastique, plastique et viscoplastique. L'identification du comportement viscoplastique est indispensable pour rendre compte des effets de fluage, de relaxation et de sensibilité à la vitesse de déformation. Deux types d'approche peuvent être considérés pour traduire en équations le comportement cyclique des monocristaux.

Une approche purement macroscopique, de type phénoménologique, a été développée, en France, par J-L. Chaboche [21] sur la même base que celle des lois de comportement des polycristaux isotropes. L'adaptation se fait en introduisant un critère d'anisotropie dans la limite élastique et en remplaçant les coefficients de durcissement cinématique non linéaire par des tenseurs d'ordre 4. Dans ceux-ci, la symétrie cubique et l'hypothèse d'incompressibilité plastique ramènent à seulement deux coefficients indépendants le nombre de paramètres à identifier, ce qui se fait par des essais de sollicitation dans deux directions de chargement $<001\rangle$ et $\langle 111\rangle$ : C'est ainsi que D. Nouailhas [22] a pu écrire il y a 3 ans une loi de comportement en viscoplasticité cyclique et anisotrope relativement simple applicable à l'AM1, décrivant de façon assez satisfaisante les évolutions $\left(\sigma, \varepsilon_{\mathrm{p}}\right)$ pour différents chargements de fatigue mécanique dans la plage de température de 650 à $1000^{\circ} \mathrm{C}$. Une illustration de la conformité de ce modèle à la réalité expérimentale est présentée à la figure 10 .

Une approche plus ambitieuse est utilisée par G. Cailletaud avec le groupe de simulation numérique du Centre des Matériaux de l'Ecole des Mines de Paris. Cette approche dite "micro-macro" consiste [23] à écrire les équations qui, dans chaque système $\mathrm{S}$ de glissement octaédrique et cubique, relient la cission $\tau^{\mathrm{s}}$ à la vitesse de glissement $\dot{\gamma}_{\mathrm{v}}^{\mathrm{s}}$ par l'intermédiaire de deux coefficients, respectivement d'écrouissage isotrope $\mathrm{r}^{\mathrm{S}}$, et d'écrouissage cinématique $\mathrm{x}^{\mathrm{S}}$. Selon le schéma de la figure 11 , ces cissions sont reliées à la contrainte macroscopique $\sigma$ par le tenseur d'orientation $\mathrm{m}^{\mathrm{s}}$,

$$
\tau \mathrm{S}=\mathrm{m} \mathrm{s}: \sigma
$$

de même que la vitesse de déformation macroscopique viscoplastique $\dot{\varepsilon}_{\mathrm{v}}$ est reliée à la vitesse de glissement,

$$
\dot{\varepsilon}_{\mathrm{v}}=\sum_{\mathrm{s} \in \mathrm{G}} m^{s} \dot{\gamma}_{\mathrm{v}}^{s}
$$

Les équations d'évolution des variables d'écrouissage de chaque système sont définies par un potentiel de dissipation fondé sur la théorie du potentiel viscoplastique de J. Rice [24]. L'intensité du glissement et la dépendance entre les différents systèmes provient du choix de la surface d'écoulement, défini par l'ensemble des expressions suivantes:

$$
\left|\dot{\gamma}_{\mathrm{v}}^{\mathrm{s}}\right|=\left[\frac{\left(\tau^{s}-x^{s}\right)-\mathrm{r}^{s}}{K}\right]^{\mathrm{n}}
$$

où $\mathrm{K}$ et $\mathrm{n}$ sont deux coefficients qui caractérisent la viscosité du matériau, et de la matrice d'interaction des différents système de glissement. 
Le système Sidolo qui regroupe les modules d'intégration numérique permettant l'expression de la loi de comportement, et le code Zébulon qui est le code de calcul 3D par éléments finis développés par la même équipe de G.Cailletaud permettent le dimensionnement des pièces. La loi de comportement a été identifiée pour l'AMl entre 20 et $1150^{\circ} \mathrm{C}$ et implantée dans le code Zébulon. A titre d'exemple la figure 12 montre le résultat du calcul des déformations viscoplastiques en surface d'une éprouvette de fatigue oligocyclique en AM1. Les traces d'émergence des plans de glissement sur les éprouvettes expérimentales confirment la validité de ces cartographies et les écarts entre les déformations mesurées et calculées sont inférieurs à $10 \%$.

Cette approche "micro-macro" ne prend pas en compte les phénomènes comme le mâclage mécanique et la montée des dislocations par diffusion des lacunes. Cela se traduit pas un peu de "raideur" des extrémités des boucles de fatigue (figure 13). Néanmoins, avec des moyens de calcul raisonnables, et la possibilité d'une adaptation de la loi de comportement aux limites physiques des mécanismes de déformation en jeu, on dispose là d'un excellent moyen de calcul du comportement des aubes de turbine à pales refroidies dans tout leur domaine de fonctionnement.

\section{SUPERALLIAGES COULES FORGES}

L'analyse du processus qui a conduit SNECMA à une mise sous contrôle stricte des performances des disques annulaires de turbine basse pression en Inco 718 permet de mettre en exergue tout le parti qui est aujourd'hui tiré du logiciel FORGE 2 [25] qu'elle a fait développer par le CEMEF entre 1983 et 1986.

\section{Dimensionnement en fatigue et taille de grain}

La durée de vie des disques de turbine b.p. est déterminée par leur résistance à la fatigue oligocyclique à laquelle ils sont soumis, du fait des efforts centrifuges en rotation entre le démarrage et l'arrêt du moteur. En effet, les températures atteintes en régime permanent dans la plage de 300 à $600^{\circ} \mathrm{C}$, font que la tenue en fluage ne constitue pas la sollicitation déterminante leur durée de vie.

Pour de tels fonctionnements à des températures modérées, les sollicitations de fatigue oligocyclique sont donc dimensionnantes et les durées de vie d'éprouvettes soumises à des cycles sinusoïdaux à des fréquences de l'ordre de $1 \mathrm{HZ}$ sont représentatives de la durée de vie des pièces. C'est ainsi que l'on démontre que l'amélioration de cette résistance à la fatigue oligocyclique passe par une réduction de la taille du grain métallurgique, comme on peut le voir sur le tableau ci-dessous établi pour des microsctrutures homogènes. 


\begin{tabular}{|c|c|c|c|}
\hline Spécifications & premium quality & grains fins & direct aged \\
\hline $\begin{array}{c}\text { taille de grains } \\
\text { ASTM }\end{array}$ & $4-6$ & $8-10$ & $10-12$ \\
\hline $\begin{array}{c}\text { Traitement } \\
\text { thermique }\end{array}$ & $\begin{array}{c}955^{\circ} \mathrm{C} / 1 \mathrm{~h} / \mathrm{air} \\
+72 \mathrm{O} / 62 \mathrm{O}^{\circ} \mathrm{C}\end{array}$ & $\begin{array}{l}955^{\circ} \mathrm{C} / 1 \mathrm{~h} / \mathrm{air} \\
+72 \mathrm{O} / 62 \mathrm{O}^{\circ} \mathrm{C}\end{array}$ & $72 \mathrm{O} / 62 \mathrm{O}^{\circ} \mathrm{C}$ \\
\hline $\begin{array}{c}\text { contrainte pour } \\
\text { rupture à } 55 \mathrm{O}^{\circ} \mathrm{C} \\
\text { en 30OOO cycles }\end{array}$ & $535 \mathrm{MPa}$ & $700 \mathrm{MPa}$ & $805 \mathrm{MPa}$ \\
\hline
\end{tabular}

Il a été depuis longtemps démontré [26] que les ruptures de fatigue s'amorcent sur les grains les plus grossiers lorsque leur répartition de taille est hétérogène. La microstructure recherchée en vue de l'allégement des disques est donc une structure homogène à grains fins. On n'abordera pas ici le problème de l'amorçage éventuel sur un carbure primaire dans les structures de grains les plus fines, qui relève de considérations sur l'élaboration et le forgeage primaire des billettes.

\section{Forgeage à grains fins de l'INCO 718}

Le forgeage des disques se fait généralement en plusieurs séquences successives de refoulement d'un lopin, perçage, expansion et matriçage à partir d'une billette de taille de grain ASTM 4 à 6 . L'obtention des microstructures à grains fins résulte de températures de forgeage basses selon l'évolution schématique représentée à la figure 14 établie par P.E. Mosser [27]. Cependant la température de forgeage ne peut être diminuée en deçà d'une certaine limite et ceci pour des raisons tenant à la raideur du matériau, qui rend ardues les opérations de forge, mais aussi aux mécanismes de transformation métallurgique associés à l'affinement du grain.

En effet, le diagramme de la figure 15 qui montre selon G. Camus [28] l'évolution de la résistance à l'écoulement en fonction de la température et de la vitesse de déformation est rapide en fonction de ces deux paramètres et il n'est pas envisageable de dépasser des efforts de 300 à $400 \mathrm{MPa}$ sans mettre en danger l'intégrité des outillages, voire, au pilon, l'engin de forge lui-même.

Par ailleurs, au fur et à mesure que l'on réduit la température de forgeage, les courbes d'évolution de la contrainte d'écoulement s'infléchissent, traduisant une hétérogénéité progressive de la déformation, qui se cantonne dans un nombre limité de bandes de glissement, et la disparition du phénomène de recristallisation dynamique. L'étendue de ce phénomène est décrit sous un autre aspect à la figure 16 qui représente la fraction recristallisée en fonction de la déformation et de la température au cours d'essais de torsion. Ceci impose, pour l'obtention de structures homogènes de grain très fins, de concevoir un traitement thermomécanique en deux opérations successives d'ébauche et de finition ménageant chacune un taux de déformation suffisant. Il est alors important lors de la deuxième opération de forgeage de ne 
pas grossir le grain recristallisé lors de l'opération précédente. Ceci est d'autant plus difficile que le grain est fin comme le montre l'équation de recristallisation

$$
\mathrm{dD}^{2} / \mathrm{dt}=\mathrm{K}(\mathrm{T})
$$

D'après cette équation le temps de croissance de l'indice ASTM $N+1$ à l'indice $\mathrm{N}$ est 4 fois plus court que le temps de croissance de l'indice $\mathrm{N}$ à l'indice $\mathrm{N}-1$.

Pratiquement, pour conserver lors des chauffages ultérieurs une taille de grains inférieure à ASTM 8, la figure 14 montre que le forgeage doit être effectué à une température inférieure à $1025^{\circ} \mathrm{C}$. La figure 17 qui représente une version à peu près réaliste du diagramme TTT de l'alliage montre que l'on entre alors dans le domaine de précipitation des durées de maintien en température compatibles avec les opérations de forge. Il est alors commode de bloquer la migration des joints de grains par une précipitation de la phase $\delta$. On voit que celle-ci intervient lors de maintiens d'une durée de vie de l'ordre de l'heure entre 950 et $1000^{\circ} \mathrm{C}$. Cette phase de composition $\mathrm{Ni}_{3} \mathrm{Nb}$ orthorombique est la forme stable de la phase $\gamma^{\prime \prime}$ précipite selon une cinétique et une morphologie (figure 18) que dépendent de l'état d'écrouissage et qui, en tous cas, sous forme de nodules finement répartis, ne modifie pas les principales caractéristiques mécaniques du matériau. Seule une précipitation trop abondante peut avoir des effets visibles car elle conduit à réduire le volume de précipitation de la phase durcissante $\gamma^{\prime \prime}$ lors du traitement de revenu. Précipitée sous forme de plaquettes elle est l'indice d'un maintien trop prolongé en four de forge et est la cause d'un délai résiduel réduit d'apparition de phases compactes (laves à haute température, $\alpha \mathrm{Cr}$ ou $\sigma$ à plus basse température). Néanmoins, en règle générale, son effet est négligeable à des fractions volumiques inférieures à quelques pourcents. Les gammes de forgeage à grains fins utilisent donc maintenent de façon systématique cette interaction entre la précipitation de $\delta$ et la stabilisation de la recristallisation pour régler la taille de grains.

\section{Modélisation du forgeage}

La compréhension des mécanismes de déformation et de précipitation conduit généralement à une maîtrise qualitative des traitements thermomécaniques qui permet en deux ou trois itérations de trouver une gamme praticable. Le délai pour une itération peut atteindre 6 mois s'il faut attendre la sanction des essais de fatigue. Cette méthode trouve actuellement sa première limite car le client motoriste demande, dans un délai bref, une gamme proche de l'optimum dès la première pièce livrée. Une deuxième limite apparaît quand la forme ou la masse de la pièce sont loin de l'expérience déjà engrangée.

Pour s'affranchir de cette limite, SNECMA s'appuie systématiquement sur le logiciel FORGE2, qui est maintenant utilisé courament par le service méthode de la forge. Ce logiciel décrit les écoulements de forgeage et l'histoire thermomécanique en tout point de la pièce forgée depuis la sortie du four jusqu'au retour à température ambiante. Dans une première étape, il permet de vérifier la capacité de la gamme à remplir les gravures sans dépasser l'effort maximum disponible sur l'engin de forge [29]. 11 permet aussi de choisir les conditions de forgeage de telle sorte que le mécanisme mis en jeu au cours des grandes déformations soit bien la recristallisation dynamique, et d'adapter les formes pour obtenir le niveau de déformation nécessaire à la recristallisation complète.

Il lui a été adjoint un module de prévision de la microstructure qui, à partir de l'histoire thermomécanique, calcule la fraction recristallisée et la taille de grain recristallisé. Ce module a été programmé par SNECMA à partir des lois de recristallisation déterminées sur échantillons rhéologiques, puis ajusté aux résultats obtenus sur pièces réelles. L'ajustement a 
essentiellement consisté en une amélioration de la description des conditions de forgeage plutôt qu'en un recalage des coefficients.

La figure 19 montre la répartition de la taille de grain et de la fraction recristallisée dans un tourillon forgé selon une spécification de type p.q. (ASTM 4-6). La figure 20 montre l'homogénéité de taille de grain à laquelle on arrive dans une pièce forgée selon une spécification de type grain fin (ASTM 8-10). Ce type de prévision directement donnée sous une forme métallurgique se révèle indispensable car il permet une évaluation directe avec un élément de la spécification métallurgique de la pièce. La capacité des logiciels tels que FORGE2 à supporter les modélisations métallurgiques justifie les efforts développés depuis 10 ans par SNECMA en simulation des procédés. L'axe de développement majeur est actuellement la modélisation des transformations métallurgiques que l'on peut rencontrer au cours des cycles thermomécaniques des différents cas de mise en oeuvre des superalliages.

\section{SUPERALLIAGES DE LA METALLURGIE DES POUDRES PREALLIEES}

Pour les disques de turbine à haute pression où la charge centrifuge est plus élevée, du fait du volume et donc de la masse des aubes, on ne peut se contenter de disques annulaires, et les formes dessinées sont plus massives, ce qui accroît d'autant leur inertie thermique, et, par voie de conséquence les sollicitations de fatigue oligocyclique. Après avoir utilisé les versions à grains fins des alliages coulés-forgés les plus raides, les progrès des turbines haute pression ont été recherchés par les concepteurs dans l'utilisation des superalliages de la métallurgie des poudres préalliées. Dans la mise en oeuvre de ces matériaux à haute résistance, le problème essentiel des concepteurs est celui de la prévision de la durée de vie sûre des pièces, problème qui est d'autant plus délicat que la limite élastique est élevée et que la taille du défaut local entraînant la ruine rapide est petit.

Deux démarches parallèles ont donc été entreprises pour fonder les modèles de prévision de durée de vie :

- l'une, qui consiste à identifier les sites d'amorçage de fissures de fatigue, ainsi que la nature et la distribution statistique des inclusions en cause, en relation avec les techniques de production du matériau,

- l'autre, qui a trait à l'étude des processus de fissuration en fonction des conditions de température, contrainte et atmosphère imposées à la pièce d'une part, et de la microstructure du matériau d'autre part.

Les matériaux supports de ces études ont été, en comparaison à l'Inco 718 coulé et forgé à grains fins, l'Astroloy, puis le N 18 [29,30] élaborés par métallurgie des poudres sous la forme de matériaux extrudés et forgés en régime isotherme, selon la route de production industrielle retenue par SNECMA.

\section{Rôle des inclusions dans l'amorçage des fissures en fatigue}

L'examen des sites d'amorçage des fissures de fatigue oligocyclique montre que, aux contraintes et aux températures envisagées pour les pièces, les carbonitrures primaires sont, 
pour une faible proportion des cas, à l'origine de la ruine de l'Inco 718 à grains fins (figure 21), mais que dans les matériaux MdP, les inclusions et les porosités sont systématiquement en cause. Pour ce qui concerne l'Inco 718, S.L. Cockcroft et A. Mitchell ont fixé [31] les critères analytiques, et proposé la technique d'élaboration, qui permettrait de s'affranchir des carbonitrures primaires. Le cas des superalliages MdP est différent [32], les porosités pouvant être considérées comme des artefacts de l'amorçage des fissures sur éprouvettes, éliminables, alors que les inclusions sont des hétérogénéités constitutives du matériau. A. De Bussac et J.C.Lautridou ont proposé [33] un modèle probabiliste qui, en prenant en compte la densité de répartition des inclusions en fonction de leur taille, détermine le risque d'amorçage d'une fissure sur une inclusion de taille donnée en fonction du volume de matière sollicité (figure 22). Compte-tenu des courbes de propreté inclusionnaire déterminées expérimentalement, ce modèle introduit un effet d'échelle d'échantillonnage de grande ampleur. A partir de la probabilité d'amorçage, il permet d'accéder à une probabilité correspondante de durée de vie en se donnant une loi de propagation. Validé à l'aide d'essais de fatigue sur des matériaux ensemencés en inclusions de taille calibrée, ce modèle rend compte du fait que les amorçages internes conduisent à des durées de vie systématiquement plus longues que les amorçages de surface comme l'illustrent les courbes expérimentales de la figure 23.

\section{Propagation des fissures de fatigue}

Compte tenu des conséquences directes de la répartition de la phase durcissante sur les propriétés mécaniques, et en particulier sur la vitesse de propagation des fissures, on s'est attaché à modéliser la précipitation de $\gamma^{\prime}$ lors de la trempe des ébauches et, après le travail de thèse de F. Devy [34] sur la simulation de la trempe, l'étude des cinétiques de précipitation est complétée par un travail en cours [35] dans les laboratoires grenoblois du CEREM et du LTPCM. Outre la prévision des contraintes de trempe qui permet de fixer les conditions acceptables de traitement thermique des ébauches de disque, les résultats escomptés doivent permettre une description quantitative fine de leur microstructure.

En parallèle, G. Hochstetter et A. Pineau ont établi [36] en fonction de la microstructure locale, les courbes de fissuration d'une ébauche de disque en $\mathrm{N} 18$. Leurs résultats sont essentiels pour la prévision des durées de vie des pièces en fonction des cartes de structures issues du modèle de forge.

En faisant varier les conditions expérimentales de chargement mécanique, de température et d'atmosphère, ils ont montré que la structure de référence, obtenue à coeur de l'ébauche est beaucoup moins sensible au couplage entre les effets de fluage et d'oxydation en pointe de fissure que les microstructures obtenues en périphérie, comme l'illustre la figure 24 . A la suite des résultats de R. Molins et E.Andrieu qui avaient mis en évidence [37] un effet de seuil de la pression partielle d'oxygène (figure 25) sur la vitesse de fissuration à $650^{\circ} \mathrm{C}$ de l'Inco 718, les mêmes auteurs ont établi l'existence d'un phénomène identique pour le N 18 (figure 26) associé à une modification de la nature de la première couche d'oxyde formée à fond de fissure qui passe de $\mathrm{Cr}_{2} \mathrm{O}_{3}$ à basse pression d'oxygène à $\mathrm{Ni} \mathrm{O}$ pour les pressions élevées. Ces résultats ouvrent des perspectives très intéressantes pour la compréhension des mécanismes couplés d'endommagement local mécanique et chimique. 


\section{CONCLUSION}

Les trois domaines d'investigations passés en revue illustrent clairement l'importance des avancées effectuées récemment dans la connaissance de superalliages essentiels pour la construction des moteurs aéronautiques. La richesse et la complexité des résultats rassemblés montrent que l'intérêt des recherches sur les superalliages est loin d'être épuisé.

Ces résultats n'ont pu être acquis qu'à l'aide de dispositifs d'investigation et de machines d'essais complexes assistés par ordinateur. Cette moisson est exploitée par une démarche systématique de modélisation physique et de simulation numérique faisant appel à de puissants moyens de calcul. Tout ceci résulte d'une stratégie de recherche concertée par SNECMA et maintenue sur le long terme, qui fonde des espérances de gain technique très significatives sur les caractéristiques des moteurs.

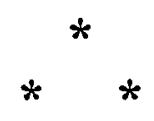

\section{BIBLIOGRAPHIE}

[1] Y. HONNORAT - les superalliages de nickel clé des technologies à hautes températures - Annales des Mines. février 1985 - p 1-10.

[2] Superalloys 19XX - Proceedings of the International Symposia held in Seven Springs Mountain Resort, Champion, Pennsylvania - Publications of T.M.S.

[3] S. WILLEMIN, M. DURAND-CHARRE - Phase equilibria in multicomponent superalloy systems - Superalloys 1988 - TMS Publ. pp 723-732.

[4] R. BONNET, D. DAVID - Estimation des énergies de fautes intrinsèques dans les phases (Ni.Al.Ti.Cr) basées sur Ni3Al - Acta.Met 39,3 pp 329-340, Mars 1991.

[5] A. BUCHON, S. CHAMBRELAN, D. BLAVETTE - Etude à la sonde atomique de la composition des phases $\gamma$ et $\gamma^{\prime}$ et de la chimie locale des superalliages monocristallins. 2ème Conférence Nationale "Superalliages monocristallins" pp 129-140 - Nancy Nov. 1990.

[6] N. SIREDEY - Solidification de monograins base nickel : microstructure et qualité cristalline - Thèse INPL - Nancy, Déc. 1991.

[7] A. ROYER - Evolutions thermique et mécanique de la microstructure de superalliages monocristallins étudiées par diffusion centrale et diffraction des neutrons et rayons $\gamma$, U.J.F. Grenoble Juin 1993.

[8] R. BONNET, A. ATI - Mise en évidence par MET d'une phase légèrement quadratique dans le superalliage CMSX2 recuit. J. Micro. Spectro. 14, pp 169-180 - 1989.

[9] D. BELLET, P. BASTIE - Temperature dependence of the lattice parameter of the $\gamma$ and $\gamma^{\prime}$ in the nickel-based superalloy CMSX2 - Phil. Mag. B 64, 2 pp 135-152 - 1991. 
[10] R. TINTILLIER - Rapport interne Snecma - Juin 1993.

[11] R. BONNET, A. ATI - observations par MET d'interactions de dislocations aux interfaces $\gamma / \gamma^{\prime}$ d'un superalliage biphasé. Acta Met. $37 \mathrm{pp} 2153$ et suiv. (1989).

[12] B. DESCAMPS, M. CONDAT, A.J. MORTON - Asymétrie traction compression dans des superalliages base nickel - Ibid [5].

[13] J. COURBON, F. LOUCHET, M. IGNAT - Cisaillement de la phase $\gamma^{\prime}$ aux températures intermédiaires $\left(650^{\circ} \mathrm{C}\right.$ à $\left.950^{\circ} \mathrm{C}\right)$ : Cinétique et Mécanismes. Ibid [5].

[14] N. CLEMENT et al - Déformation in-situ à différentes températures de monocristaux de phase $\gamma^{\prime}$ de CMSX2. Ibid [5].

[15] R. MORBIOLI, S. ALPERINE, R. MEVREL - Tendances récentes dans la protection des aubes de turbine. 15ème Colloque des Matériaux pour l'Aéronautique et l'Espace. AAAF Publ. Juin 1993.

[16] L. REMY - Recent developments in thermal fatigue - EGF6 Mechanical Engineering Pub. pp 353-377, London - 1990.

[17] E. CHATAIGNIER, L. REMY - Influence d'une protection sur la fatigue anisotherme de l'AM1 - Rapport Contrat Snecma Janv. 1992.

[18] M. MARCHIONNI, D. GOLDSCHMIDT, M. MALDINI - High temperature mechanical properties of CMSX4 + yttrium single crystal nickel-base superalloy - Superalloys 1992 - TMS Publ. pp 775-784.

[19] P. AIMONE, R. McCORMICK - The effects of yttrium and sulphur on the oxydation resistance of an advanced single crystal nickel based superalloy - Superalloys 1992 - TMS Publ. pp 817-823.

[20] P. CARON - ONERA - Communication privée Avr. 1993.

[21] J.L. CHABOCHE - Viscoplastic constitutive equations for the description of cyclic and anisotropic behavior of metals - Bull. Acad Polonaise des Sciences Série Sc. et Tech. 25, 1977.

[22] D. NOUAILHAS - Loi de comportement en voscoplasticité cyclique et anisotrope : Application au cas des matériaux à symétrie cubique - La Recherche Aérospatiale $1990 \mathrm{n}^{\circ} 3 \mathrm{pp}$ 11-28.

[23] L. MERIC, P. POUBANNE, G. CAIlLETAUd - Single Modeling for structural calculations - Model presentation - Trans. ASME 113, pp 162-170, Janv. 1991.

[24] J.R. RICE - Inelastic constitutive relations for solids : an internal variable theory and its application to metal plasticity - J. Mech. Phys. Solids, $19 \mathrm{n}^{\circ} 6 \mathrm{pp}$ 433-455 - 1971.

[25] Y. GERMAIN - Modélisation par éléments finis d'écoulements viscoplastiques avec frottement - Application au forgeage à chaud - Thèse - Ecole Nationale Supérieure des Mines de Paris - Juin 1985.

[26] H.F. MERRICK - The low cycle fatigue of three wrought nickel-base alloys - Met. Trans. 5 pp 891-903 - Avr. 1974.

[27] P.E. MOSSER, G. LECONTE, J. LERAY, A. LASALMONIE, Y. HONNORAT Metallurgical aspects of forge modelling in Alloy 718 - Superalloy 718 TMS Publ. pp 179-188 - 1989. 
[28] G. CAMUS - Traitement thermomécanique de l'Inco 718 pour différentes applications sur turbomachines - Thèse, INP - Toulouse 1986.

[29] C. DUCROCQ, A. LASALMONIE, Y. HONNORAT - A new damage tolerant PM superalloy for high temperature turbine disks - Superalloys 88 - TMS Publ. pp 63-72.

[30] J.Y. GUEDOU, J.C. LAUTRIDOU, Y. HONNORAT - N18, PM superalloy for disks : development and applications - Superalloys 92 - TMS Publ. pp 267-276.

[31] S.L. COCKCROFT, T. DEGAWA, A. MITCHELL, D.W. TRIPP, A. SCHMALZ Inclusion precipitation in superalloys - Superalloys 92 - TMS 92 Publ. pp 577-586.

[32] Y. HONNORAT - Vacuum metallurgy in jet engine manufacture - 7th Int. Vacuum metallurgy Conf. - SF2M Publ. Antibes - Mai 1992.

[33] A. DE BUSSAC, J.C. LAUTRIDOU - A probabilistic model for prediction of LCF surface crack initiation in PM alloys - Fact. and Fract. of Engineering Mat. and structure. A paraître.

[34] F. DEVY - Description, modélisation et simulation de la trempe des superalliages Thèse Univ. Paris VI 1991.

[35] Contrat MRT 90A0566. 


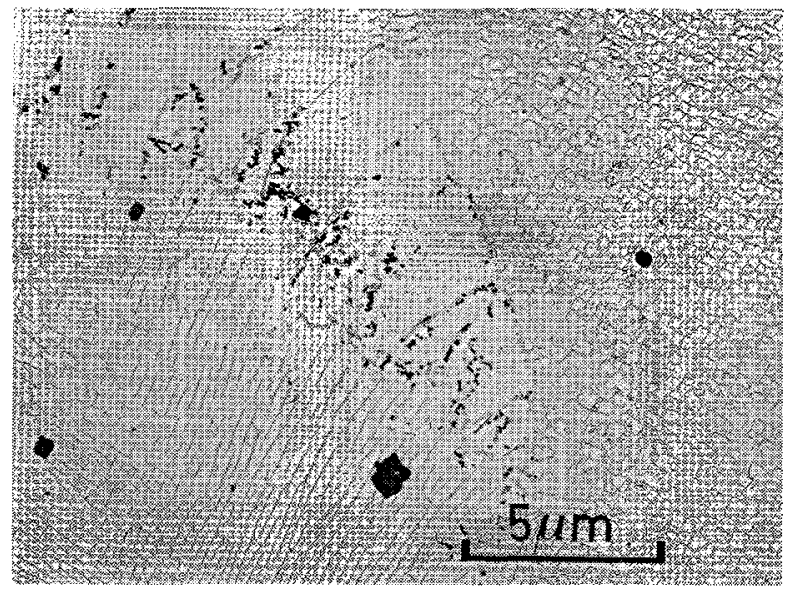

Fig. 1 Exemple de structure de grain de solidification dans une variété d'IN100 -Noter l'évolution de $\gamma^{\prime} /$ carbure vers $\gamma^{\prime} / \gamma$ -

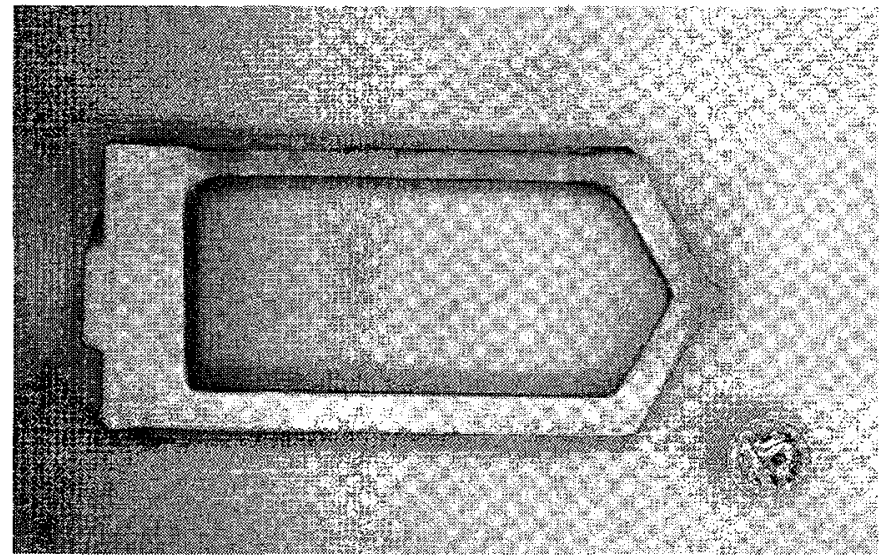

Fig. 2 Eprouvette de solidification monocristalline multi-branches en AM1 


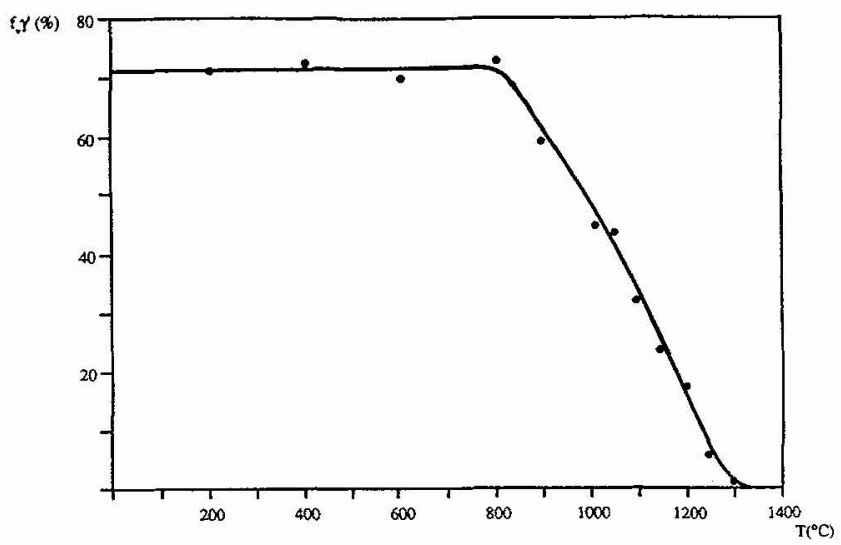

Fig. 3 AM1 - Evolution de la fraction volumique de $\gamma^{\prime}$ en fonction de la température

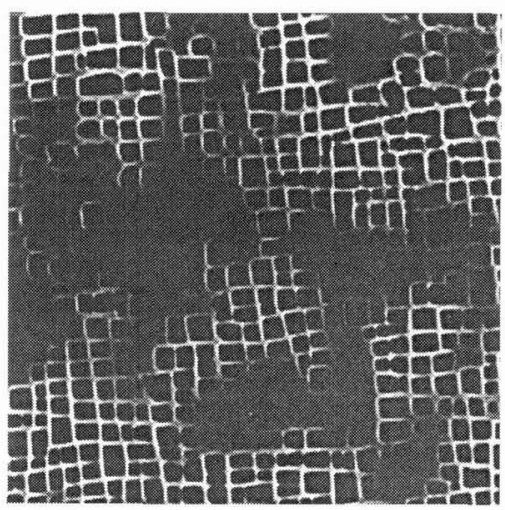

a

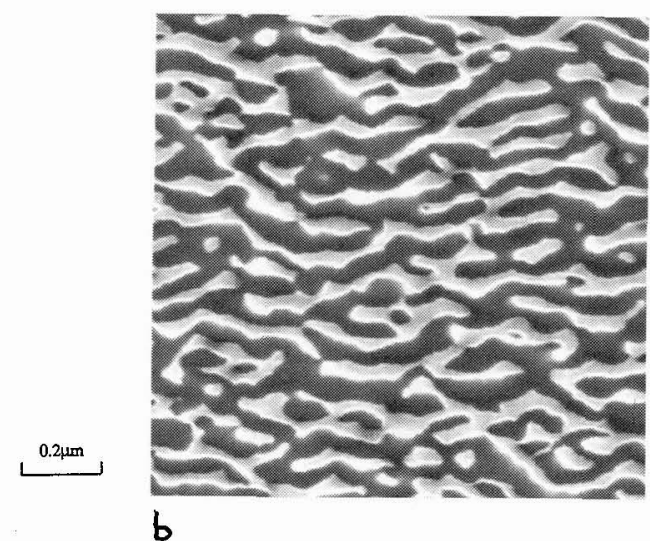

Fig. 4 Morphologie des précipités $\gamma^{\prime}$ dans l'AM1

a) Après traitement thermique complet

b) Après déformation de fluage 


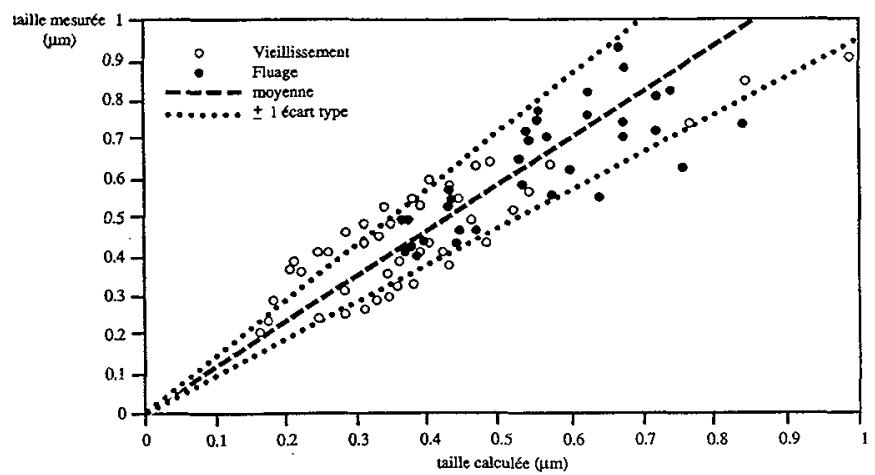

Fig. 5 AM1 - Prédictibilité de la taille des précipités $\gamma^{\prime}$ selon un modèle LSW

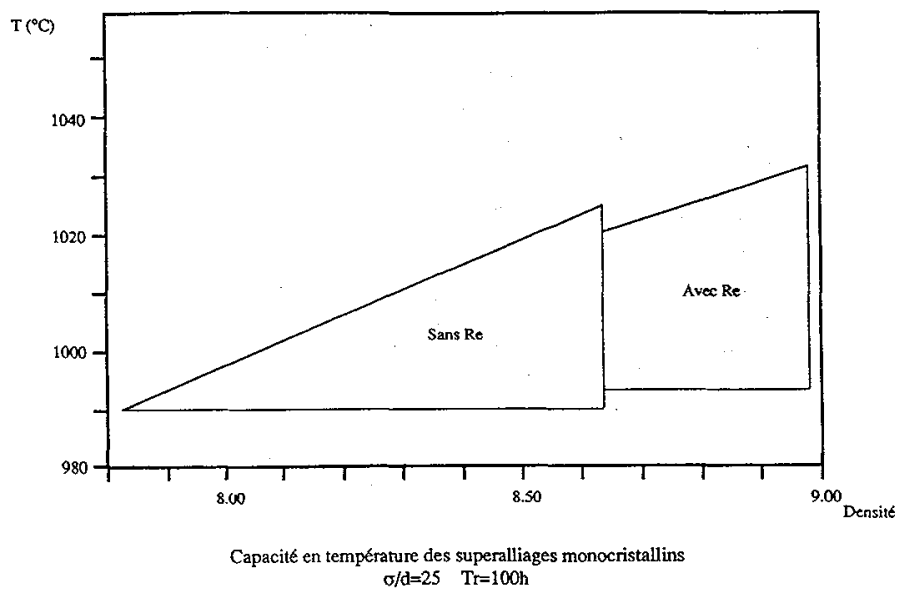

Fig. 6 Capacité en température des superalliages monocristallins $\sigma / \mathrm{d}=25 \mathrm{Tr}=100 \mathrm{~h}$ 


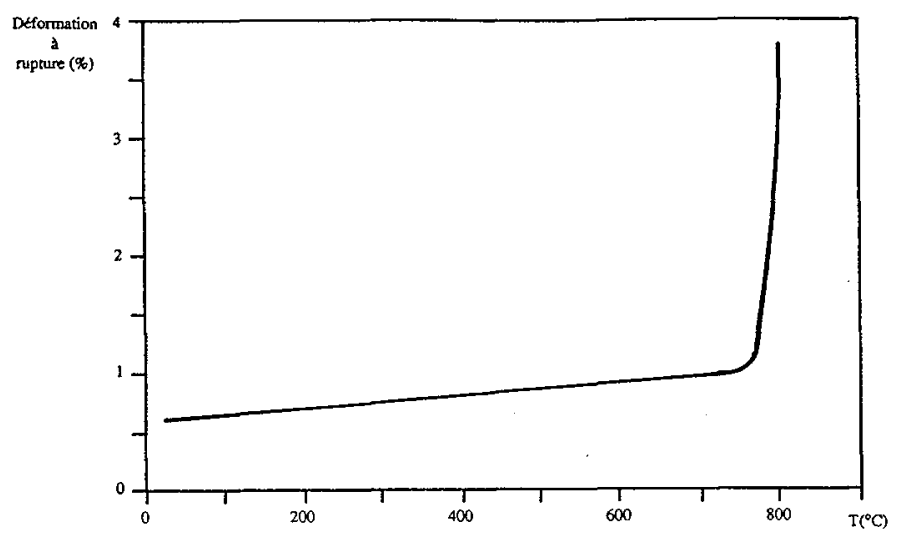

Fig. 7 Revêtement C1A - Evolution de la ductilité en fonction de la température

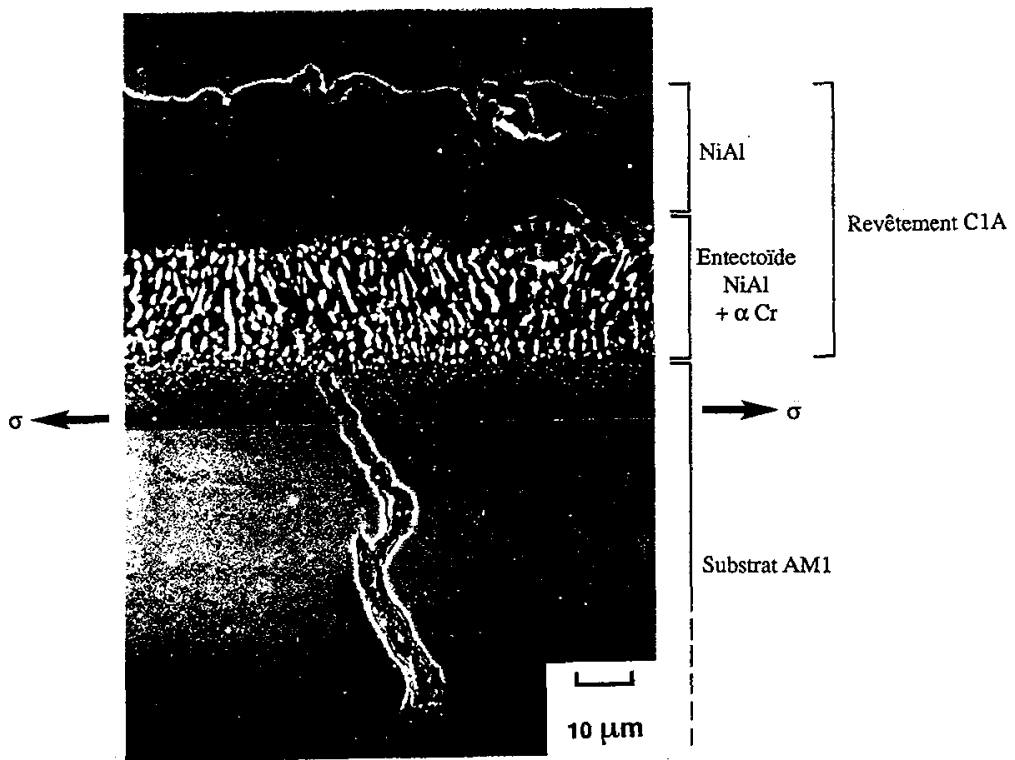

Fig. $8 \mathrm{AM} 1$ revêtu $\mathrm{C} 1 \mathrm{~A}-$ Faciès de fissuration en fatigue anisotherme entre 600 et $1100^{\circ} \mathrm{C}$ $\epsilon_{\mathrm{T}}=2 \%$ 
$\varepsilon$ inélastique (\%)

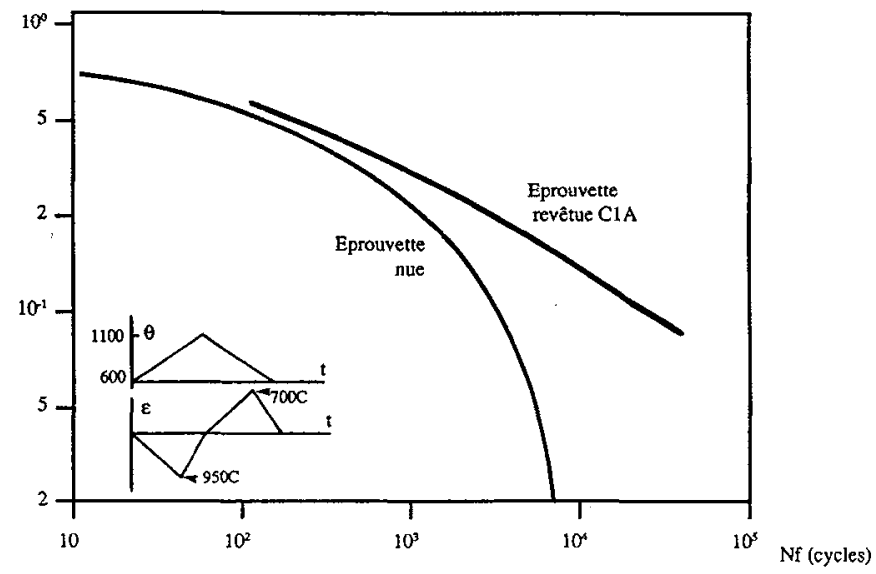

Fig. 9 AM1 - Durée de vie en fatigue anisotherme en fonction de la déformation inélastique Direction de sollicitation [001]

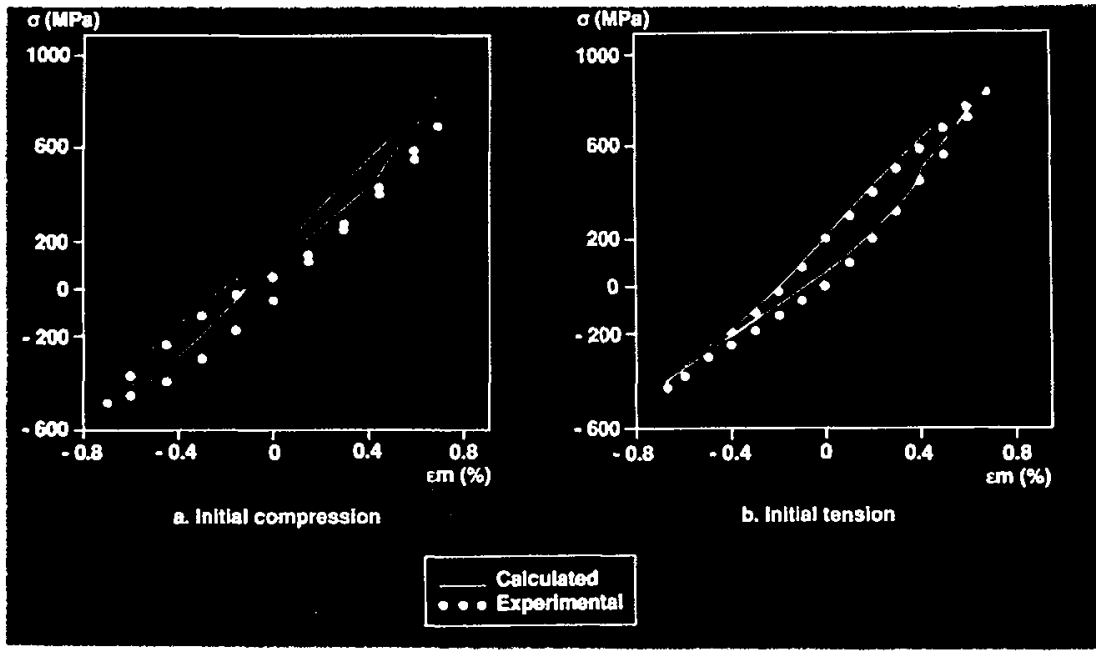

Fig. 10 Boucles de contrainte et déformation en fatigue thermomécanique dans l'AM1 a) Compression initiale

b) tension initiale 
> Elasticité macroscopique (et : $\left.\dot{\varepsilon}=\dot{\varepsilon}_{\mathrm{e}}+\dot{\varepsilon}_{v}\right)$

$>$ Loi viscoplastique avec seuil sur les systèmes de glissement

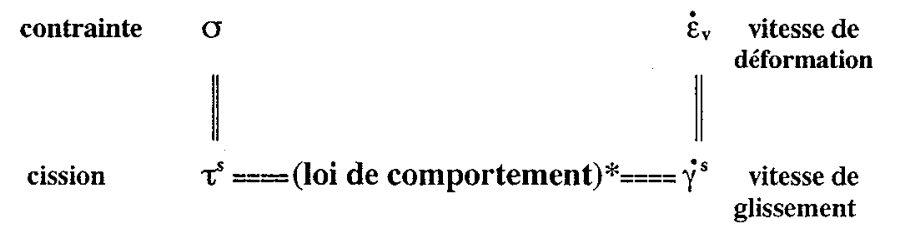

* Faisant intervenir :

- un écrouissage cinématique

- un écrouissage isotrope avec interaction entre systènes

Fig. 11 Schéma de calcul d'une loi de comportement des superalliages monocristallins selon le modèle développé par Georges Cailletaud

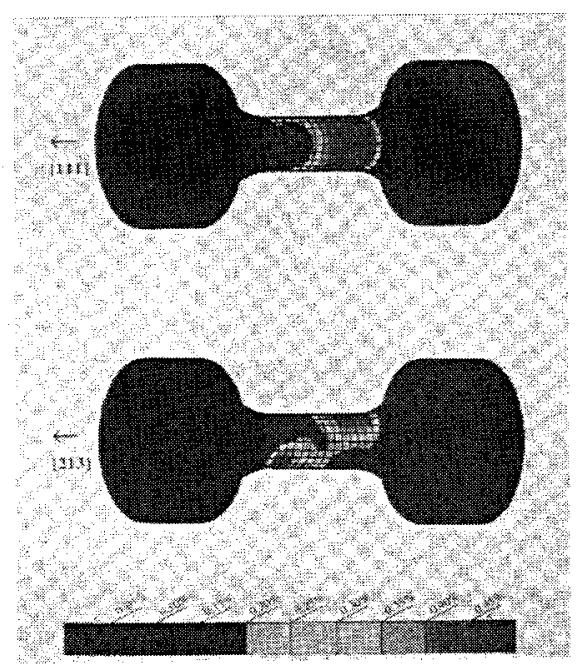

Fig. 12 Isovaleurs de déformation viscoplastique équivalente d'éprouvettes de fatigue olygocyclique en AM1

a) Cas de l'éprouvette d'axe (111)

b) Cas de l'éprouvette d'axe (213) 


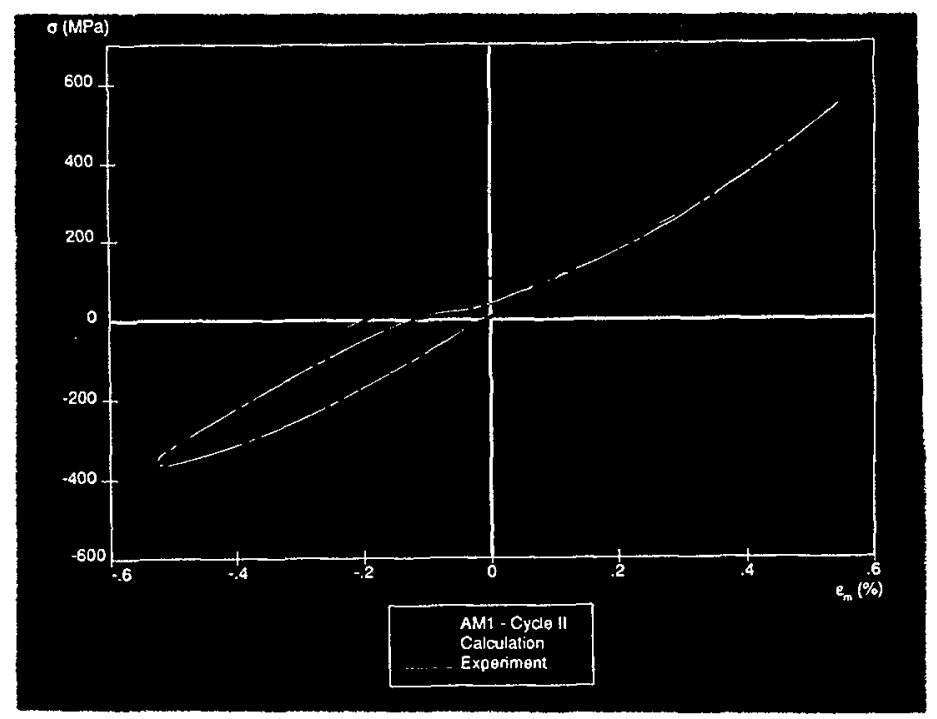

Fig. 13 Boucles de contraintes et de déformation en fatigue thermomécanique dans l'AM1

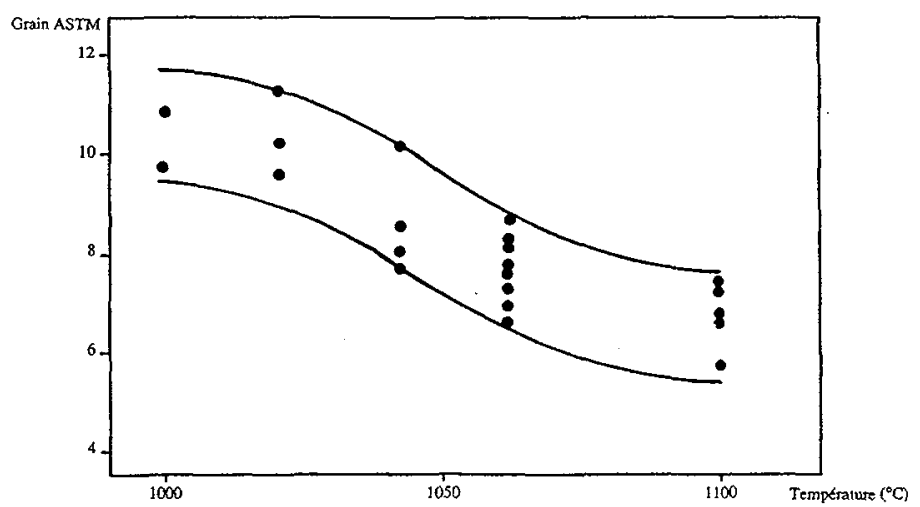

Fig. 14 INCO718 - Taille de grain en fonction de la température de forgeage 


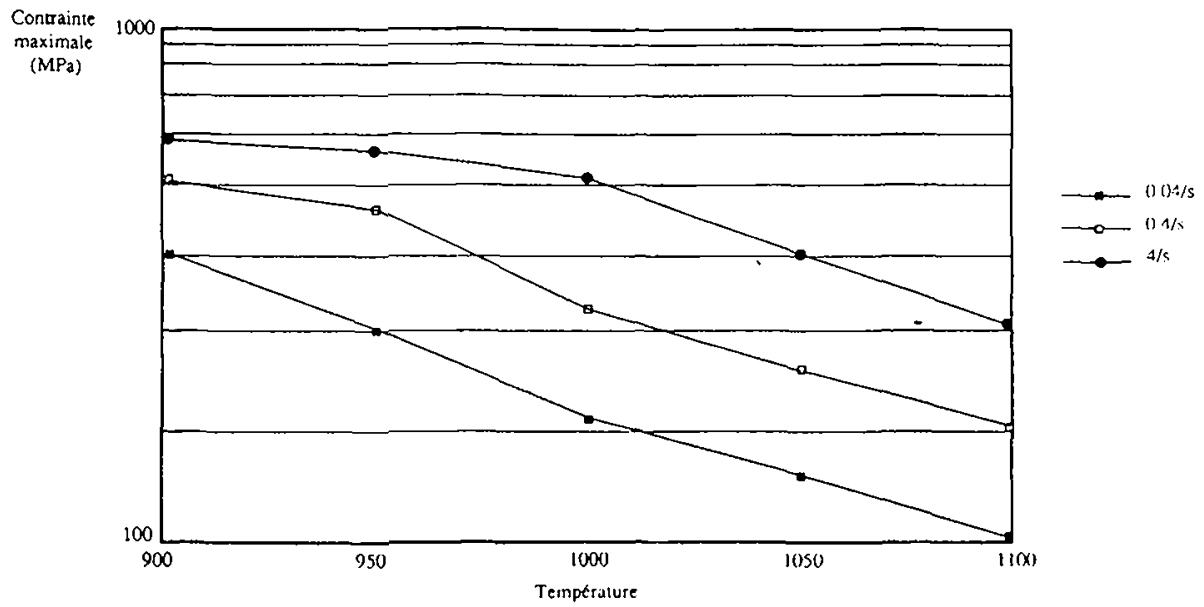

Fig. 15 INCO718 - Contrainte d'écoulement en fonction de la température

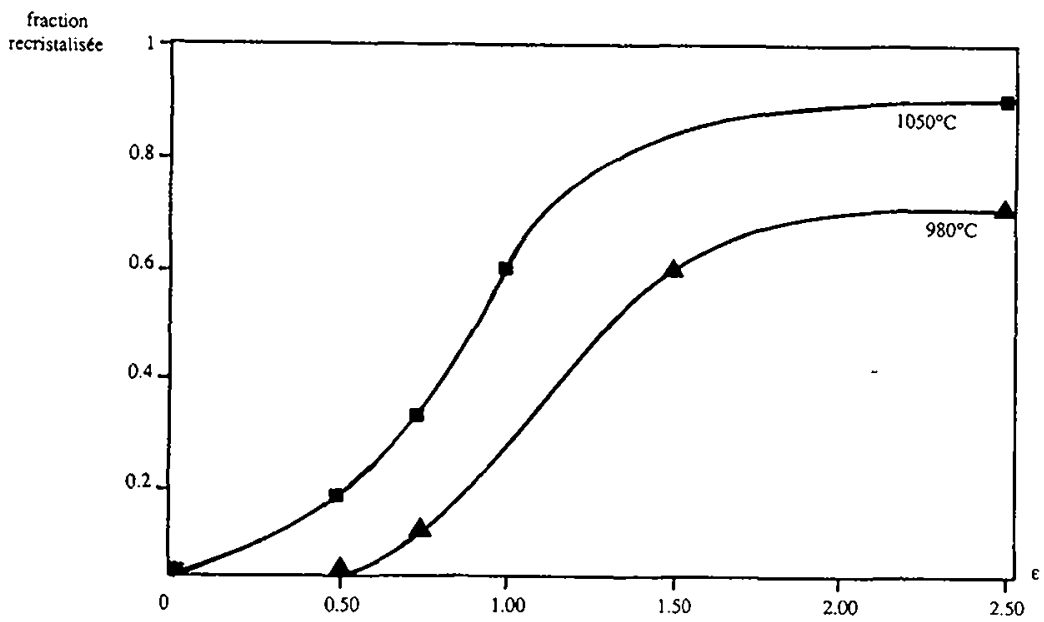

Fig. 16 INCO718 - Fraction recristalisée en fonction de la déformation, à 980 et $1050^{\circ} \mathrm{C}$ $\epsilon=3 \cdot 10^{-2} \mathrm{~S}^{-1}$ - Taille de grain initiale ASTM8 


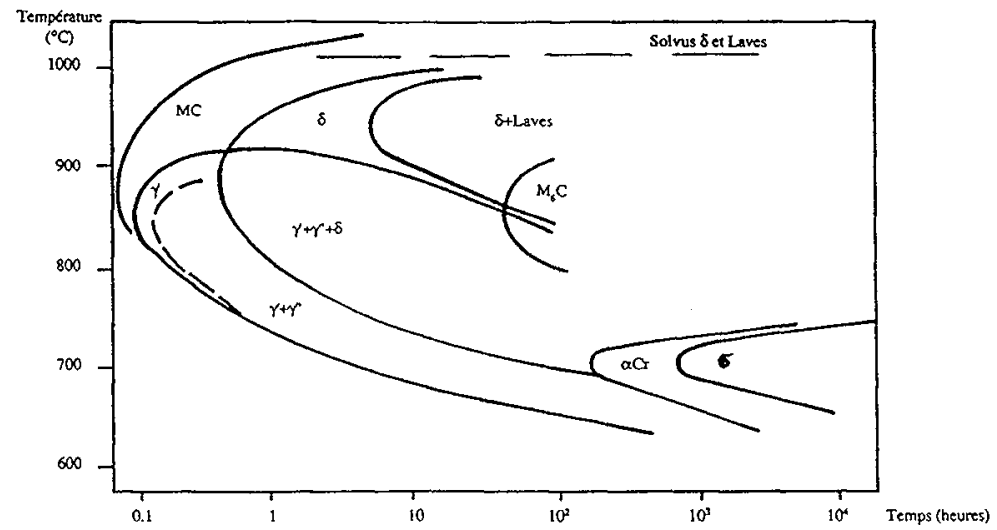

Fig. 17 INCO718 - Diagramme TTT (D'après Radavich, Boesch, Eiselstein)

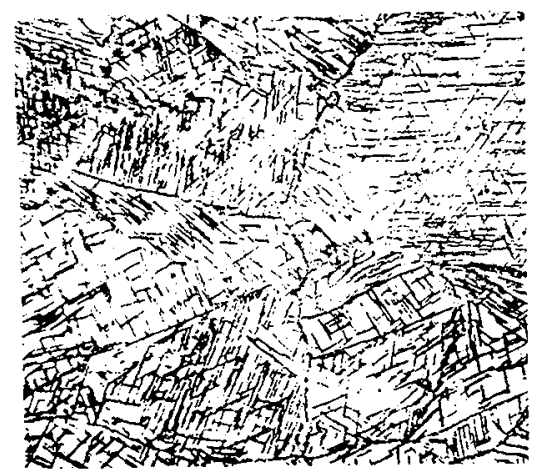

Precipitation statique en plaquettes

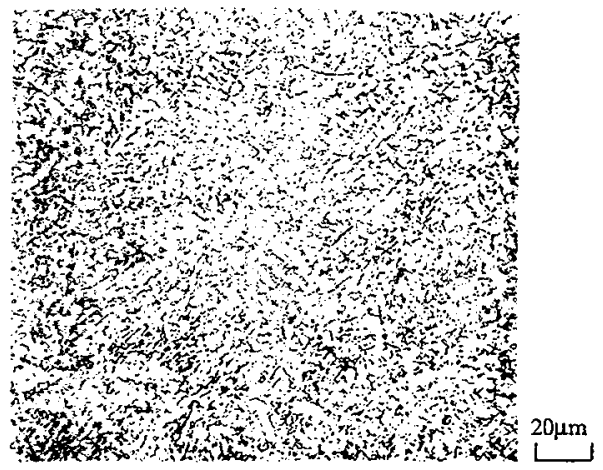

Precipitation sousdêformation en globules

Fig. 18 INCO718 - Morphologie de la phase delta 


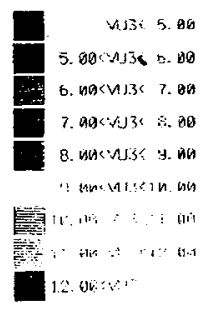

ESTIMATTON DE LA TAILLE DES GRAINS RECRISTALLISES

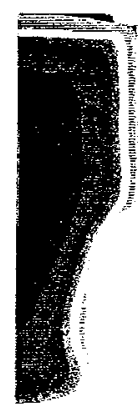

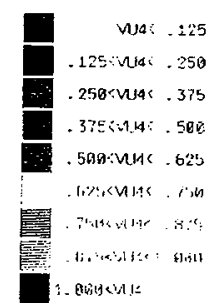

ESTIMATION DE LA FRACTION RECRISTALLISEES

Fig. 19 INCO718 - Estimation de la taille de grain et de la fraction recristallisées lors du filage de 1'ébauche du tourillon de turbine BP du moteur M88
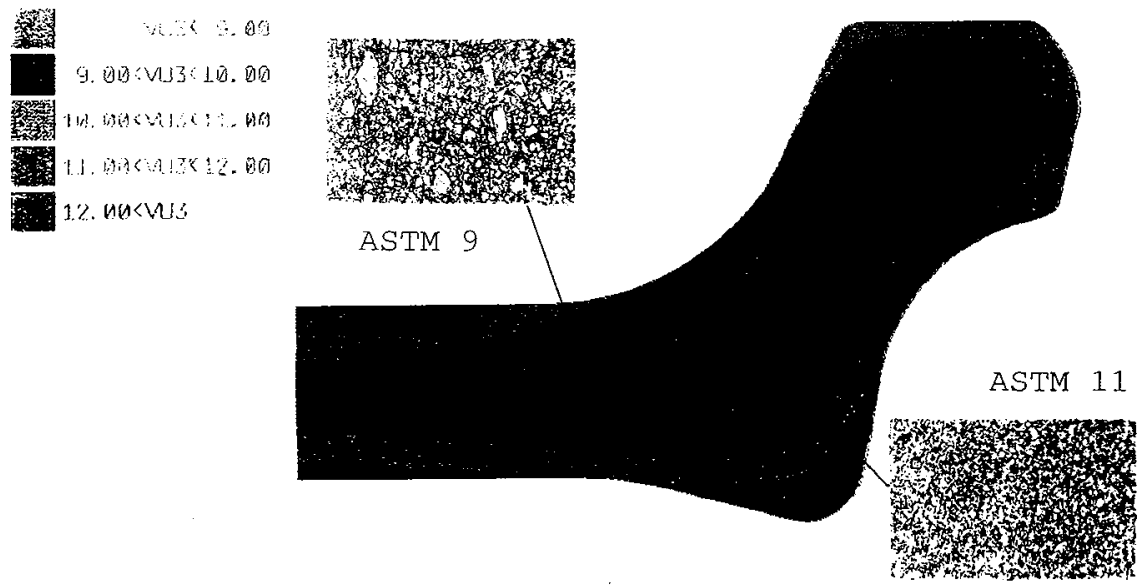

Fig. 20 INCO718 - Modélisation de la taille de grain recristallisée au matriçage d'une ébauche de disque 


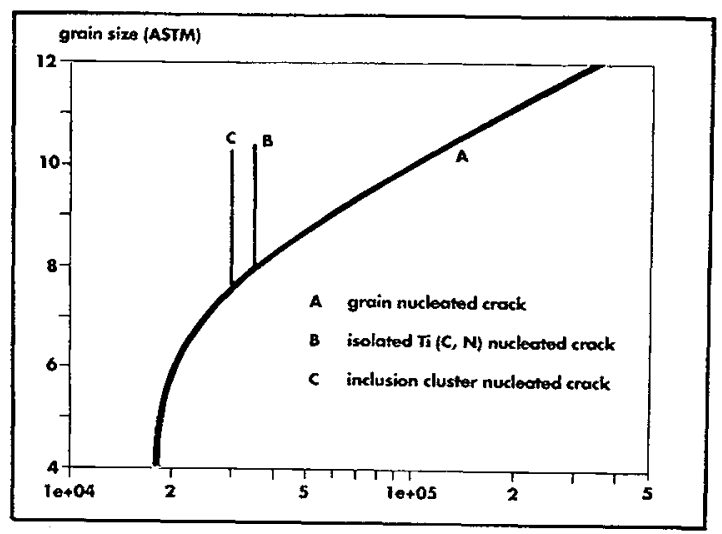

Fig. 21 Courbe du minima de durée en fatigue oligocyclique dans l'INCO718 à $350^{\circ} \mathrm{C}$ pour une amplitude de déformation de 0.62
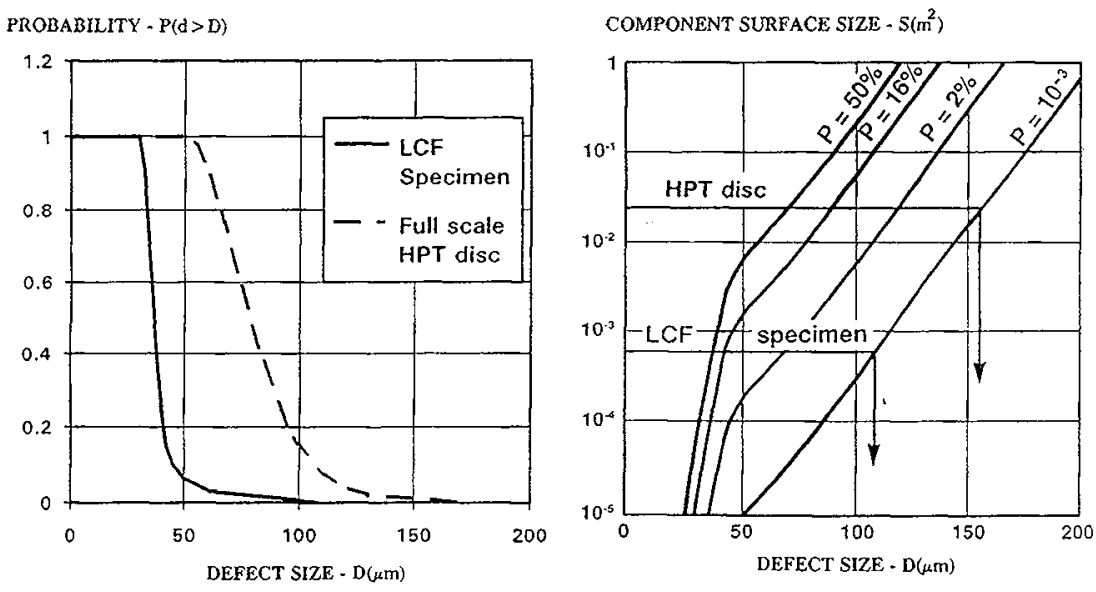

Fig. 22 Illustration de l'effet d'échelle - Matériaux standards 


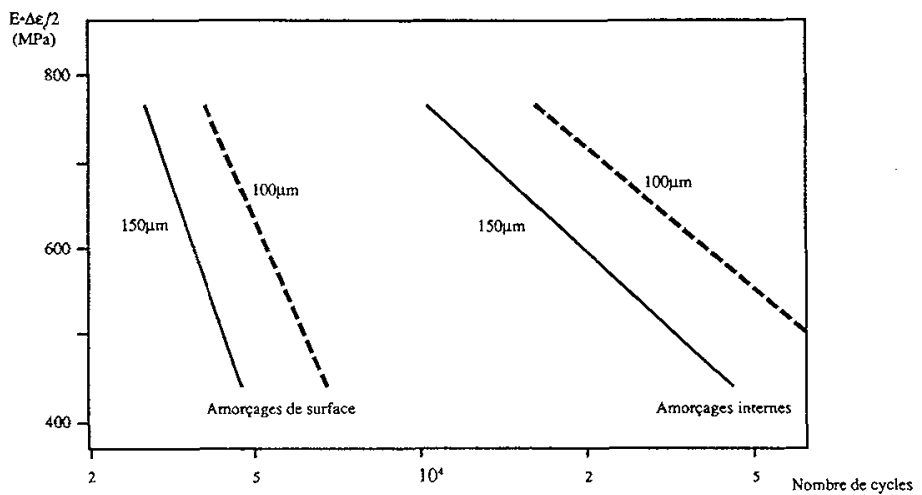

Fig. $23 \mathrm{~N} 18$ - Durée de vie en fatigue olygocyclique à $650^{\circ} \mathrm{C}$ en fonction de la taille et du site d'inclusion à l'amorçage

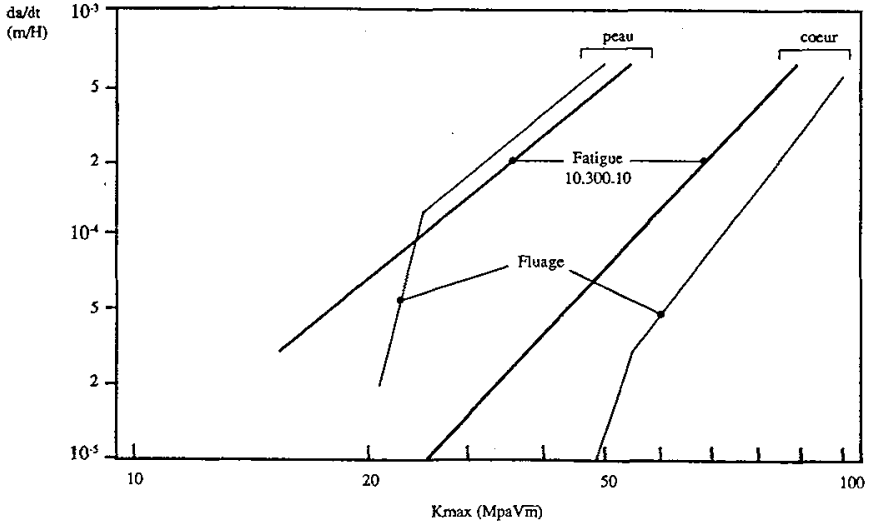

Fig. 24 N18 - Cinétique de fissuration à l'air à $650^{\circ} \mathrm{C}$ en fatigue et fluage 


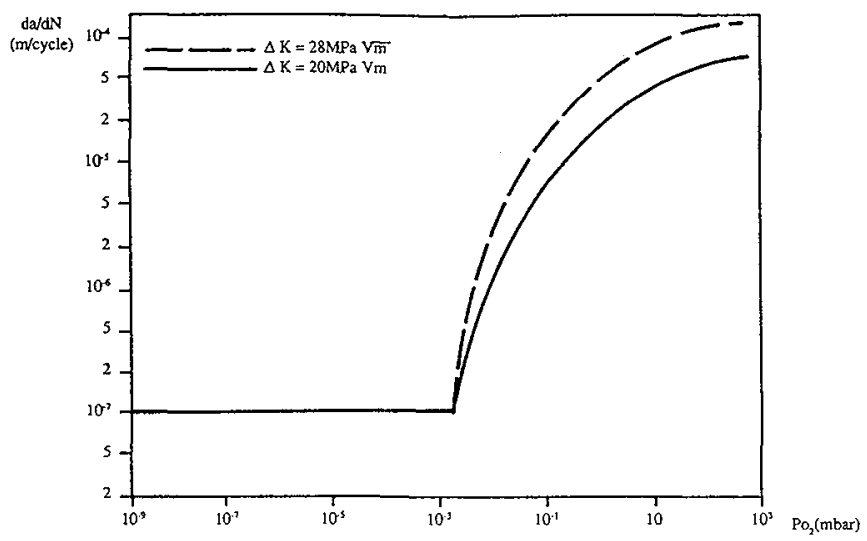

Fig. 25 INCO718 - Influence de la pression partielle d'oxygène sur la vitesse de fissuration en fatigue olygocyclique à $650^{\circ} \mathrm{C}$

Cycles trapézoïdal $\mathrm{Tm}=300 \mathrm{~S}-\mathrm{R}=0.1$

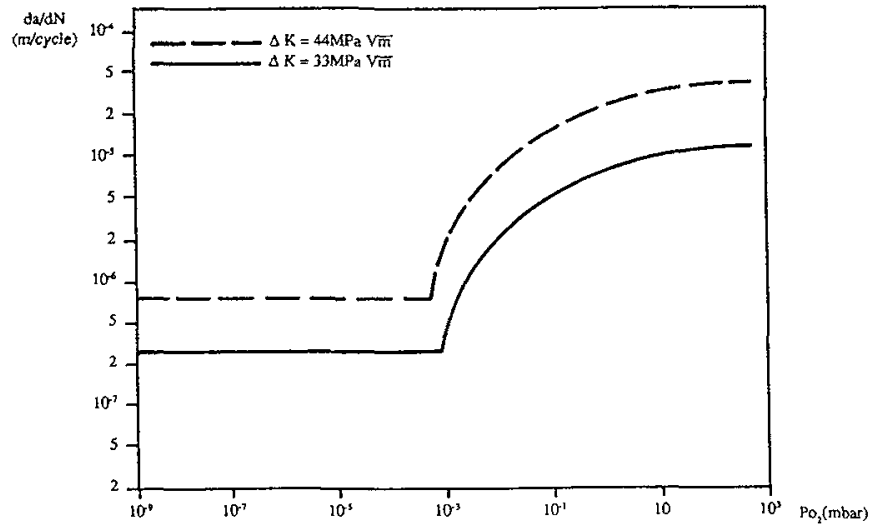

Fig. 26 N18 - Influence de la pression partielle d'oxygène sur la vitesse de fissuration en fatigue olygocyclique à $650^{\circ} \mathrm{C}$

Cycles trapézoïdal $\mathrm{Tm}=300 \mathrm{~S}-\mathrm{R}=0.1$ 